\title{
Direct Imaging Raman Microscope Based on Tunable Wavelength Excitation and Narrow-Band Emission Detection
}

\author{
G. J. PUPPELS, M. GROND, and J. GREVE* \\ Department of Applied Physics, University of Twente, P.O. Box 217, 7500 AE Enschede, The Netherlands
}

\begin{abstract}
A new type of imaging Raman microscope is described. First the advantages and disadvantages of the two possible approaches to Raman microscopy based on signal detection by means of a charge-coupleddevice camera (i.e., direct imaging and image reconstruction) are discussed. Arguments are given to show that in most cases direct imaging is to be preferred over image reconstruction, because it provides the desired information in less time. In the direct imaging Raman microscope presented in this communication, detection of scattered light occurs in a narrow interval around a fixed wavelength. Selection of the Raman wavenumber shift at which an image is recorded is established by tuning the wavelength of the exciting laser light in such a way that the wavelength of the Raman scattered light with the desired Raman shift coincides with the detected wavelength. The microscope has been incorporated in a Raman microspectrometer in a way that enables easy switching between the imaging and the multichannel spectroscopy modes of operation. Bright field, fluorescence, and Raman microscopic images can be obtained.
\end{abstract}

Index Headings: Raman microscopy; Imaging; Charge-coupled device; Dye laser; Dielectric filters; Fluorescence microscopy.

\section{INTRODUCTION}

Raman spectroscopy is a versatile vibrational spectroscopy, applied in research fields ranging from materials sciences to molecular and cell biology. Raman microspectrometers can probe samples with submicron spatial resolution, yielding information about chemical composition and molecular structure.

In inhomogeneous samples it is often important to assess the spatial distribution of molecular species or structure. Examples are the molecular composition of bands and interbands of polytene chromosomes, ${ }^{1}$ the distribution of water and proteins in eye lenses, ${ }^{2}$ and the precise subcellular location of carotenoids in cells. ${ }^{3}$ One way to address such questions is by means of Raman microscopy, i.e., imaging of an object in Raman scattered light. In a Raman microscope only a small part of the spectrum of the scattered light is used to form the image-namely, the part corresponding to a Raman shift characteristic for the type of molecule of which the spatial distribution in the sample is investigated.

Such instruments are the Raman equivalent of the well-known fluorescence microscopes. The basic difference from the instrumentational point of view is that Raman microscopy poses much higher demands with respect to the spectral bandwidth of the exciting light source, the spectral resolution of the emission detection, and the signal detection efficiency of the whole setup.

Received 20 January 1993; revision received 8 March 1993.

* Author to whom correspondence should be sent.
The more fundamental difference is of course that Raman imaging, in contrast with fluorescence imaging, provides information about the chemical composition of a sample without the need for probes or labels.

In 1975 Delhaye and Dhamelincourt were the first to construct a Raman microscope and to discuss a number of different approaches to Raman microscopy. ${ }^{4}$ Their instrument was based on a grating monochromator for the selection of the desired Raman shift and an intensified video camera for image recording.

The field of Raman imaging has seen a lot of activity lately, thanks to several technological developments. Not the least of these was the development of cryogenically cooled slow-scan charge-coupled device (CCD) cameras, which combine high quantum efficiency with virtually noiseless operation. A number of methods to achieve image formation on the basis of Raman scattered light, employing CCD-cameras for signal detection, have recently been reported. ${ }^{5-12}$ Basically two different approaches can be distinguished: direct imaging and image reconstruction. The distinction between the two lies in the choice that is made of how to deal with the problem that the Raman signal is 3-dimensional (1 spectral and 2 spatial dimensions) whereas the detector is a 2-dimensional array.

\section{ANALYSIS OF RAMAN IMAGING APPROACHES}

Direct Imaging. In direct imaging methods a sample is globally illuminated. An image of the sample is formed on the CCD chip, based on light scattered by the sample, after the desired spectral component of the scattered light is filtered out. Examples of direct imaging Raman microscopes are the instrument developed by Batchelder et al., ${ }^{5}$ employing a dielectric narrow-band transmission filter for Raman shift selection, and the instrument of Treado et al., ${ }^{6}$ which utilizes an acousto-optical tunable filter. Our own design, presented in this communication, is also based on filtering the Raman scattered light by means of a dielectric narrow-band transmission filter.

In many (e.g., biological) applications, samples are vulnerable to laser-light-induced damage. This problem limits the allowable laser light intensity on the sample. The advantage of the direct imaging method is that the total available laser power is distributed over the entire sample, so that in principle each spatially resolved position of the sample can be illuminated and analyzed simultaneously with maximum allowable laser light intensity. In the following discussion, this will be referred 
to as the power distribution advantage of the direct imaging method.

The disadvantage of direct imaging is of course that spectral information is lost. In order to obtain a Raman image at a different wavenumber shift (e.g., in order to obtain information about the spatial distribution of a second chemical component of the sample), a new measurement is necessary.

Image Reconstruction. In the image reconstruction methods, full spectral information for each point of the sample is maintained, sacrificing one dimension of spatial information. Therefore multiple measurements (as many as the desired number of pixels in this spatial dimension) have to be carried out before an image is obtained. The advantage is that, after all measurements have been made, an image of the sample can be reconstructed at any desired wavenumber shift.

Line Illumination. The simplest of these methods is line illumination of a sample and scanning the sample through this line. This approach has been used by Bowden et $a l .{ }^{7}$ and by Barbillat et $a l .^{8}$ For each scan position of the sample a Raman measurement is made. One dimension of the CCD chip is used for spatial information (along the line of illumination); the other dimension is used to record spectral information. After the whole sample is scanned through the illumination line, complete spectral information from every point of the object is obtained, and images of the sample can be generated at any desired wavenumber shift.

However, unless many images at different Raman shifts are needed, this approach is much slower than the direct imaging approach. Operating with the maximum allowable laser light intensity on the sample implies that the total laser power on the sample with the use of line illumination is lower than in the direct imaging methods by a factor equal to the ratio of the illuminated areas in both cases. Taking, for example, the situation in which 100 pixels of spatial information are needed in the sample scan direction implies that the total laser power on the sample with the use of line illumination is two orders of magnitude lower than in a direct imaging method. This, in turn, means that in order to image a sample at a particular wavenumber, signal collection times must be two orders of magnitude larger for the line illumination method than for direct imaging methods in order to arrive at the same signal-to-noise ratio.

Hadamard Transform Raman Microscopy. In a recent series of papers Treado et al. ${ }^{9-11}$ and Liu et al. ${ }^{12}$ described a Hadamard transform Raman microscope. This instrument also provides Raman images by means of image reconstruction. The sample is globally illuminated with laser light. One spatial dimension of the (2-dimensional) image of the sample is encoded with a mask (1D-multiplexing) after which the image is compressed to one dimension (a line). This line is focused onto the entrance slit of a stigmatic Raman spectrometer, as in the case of line illumination described above. Performing Raman measurements with linearly independent encodings enables one to retrieve the compressed spatial information by suitably adding and subtracting the results of the different measurements. The number of Hadamard mask elements used to encode the compressed spatial dimension determines the number of pixels that are obtained after reconstruction of this spatial dimension and determines the number of measurements needed to retrieve this information. Therefore, the same number of measurements is needed in Hadamard transform Raman microscopy as in the line illumination method in order to reconstruct an image with the same number of pixels. In both cases full spectral information is obtained for each image pixel.

It has been claimed, however, that due to the spatial multiplexing in Hadamard transform Raman microscopy, the amount of time needed for the measurements can be significantly reduced..$^{9-12}$ As in the direct imaging methods, the sample is globally illuminated, so that the total laser power on the sample can be much higher than in the line illumination method, leading to higher signal intensity levels.

However, there exists no physical ground for the claim that this method would lead to better signal-to-noise ratios and, consequently, reduce signal collection times. It is well known that when the signal-to-noise ratio in a measurement is photon-noise (shot-noise) limited, Fourier or Hadamard transform techniques do not lead to a multiplex advantage. ${ }^{13,14}$ In other words, under conditions of photon-noise-limited signal detection, consecutive scanning of channels yields the same signal-to-noise ratio in the same amount of time as would be obtained by multiplexing the channels. This point applies of course to spatial as well as to spectral multiplexing.

In the Hadamard transform Raman microscope, 1-dimensional spatial multiplexing is applied and a cryogenically cooled CCD camera is used for signal detection. ${ }^{9-12}$ Signal detection by such a camera is essentially photon-noise limited because the amount of detector noise added to the signal is negligible in virtually any situation (dark current is of the order of 1 electron per pixel per hour; read-out noise in the models commercially available at this moment can be as low as 3 to 4 electrons or less, 1 electron equalling 1 detected photon). Therefore the spatial multiplexing would not be expected to lead to an improvement in signal-to-noise ratio (or reduction of signal collection times). This observation is illustrated below.

Comparison of Signal-to-Noise Performance of Raman Imaging Approaches. Due to the very low signal levels usually encountered in Raman experiments, the single most important criterion for the choice of a particular method is the total signal collection time needed to obtain an image of sufficient signal-to-noise ratio.

Below we derive expressions for the signal-to-noise ratio obtained for direct imaging Raman microscopy, linescanning Raman microscopy, and Hadamard transform Raman microscopy. These derivations will be based on a simple model and will serve to make a quantitative comparison of the performance of the different methods under different experimental conditions.

Model. Assume that one wants to measure the intensity of the Raman signal at $M$ different wavenumber shifts for the $N$ pixels of a 1-dimensional object by means of a 1-dimensional detector array. (This situation of 1-D Raman imaging by means of a $1 \mathrm{D}$ detector is of course identical to the case of $2 \mathrm{D}$ Raman imaging by means of a $2 \mathrm{D}$ detector. In both cases one spatial dimension or the spectral dimension has to be either scanned or encoded. 
OBJECT

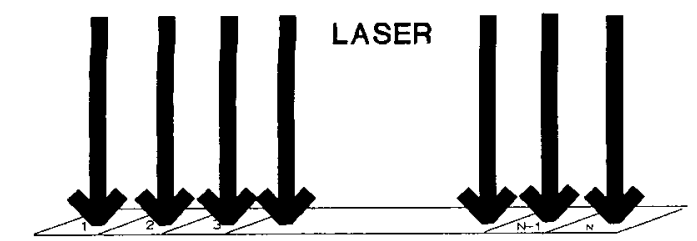

$\triangle \mathrm{CM}-1$ SELECTION

IMAGE
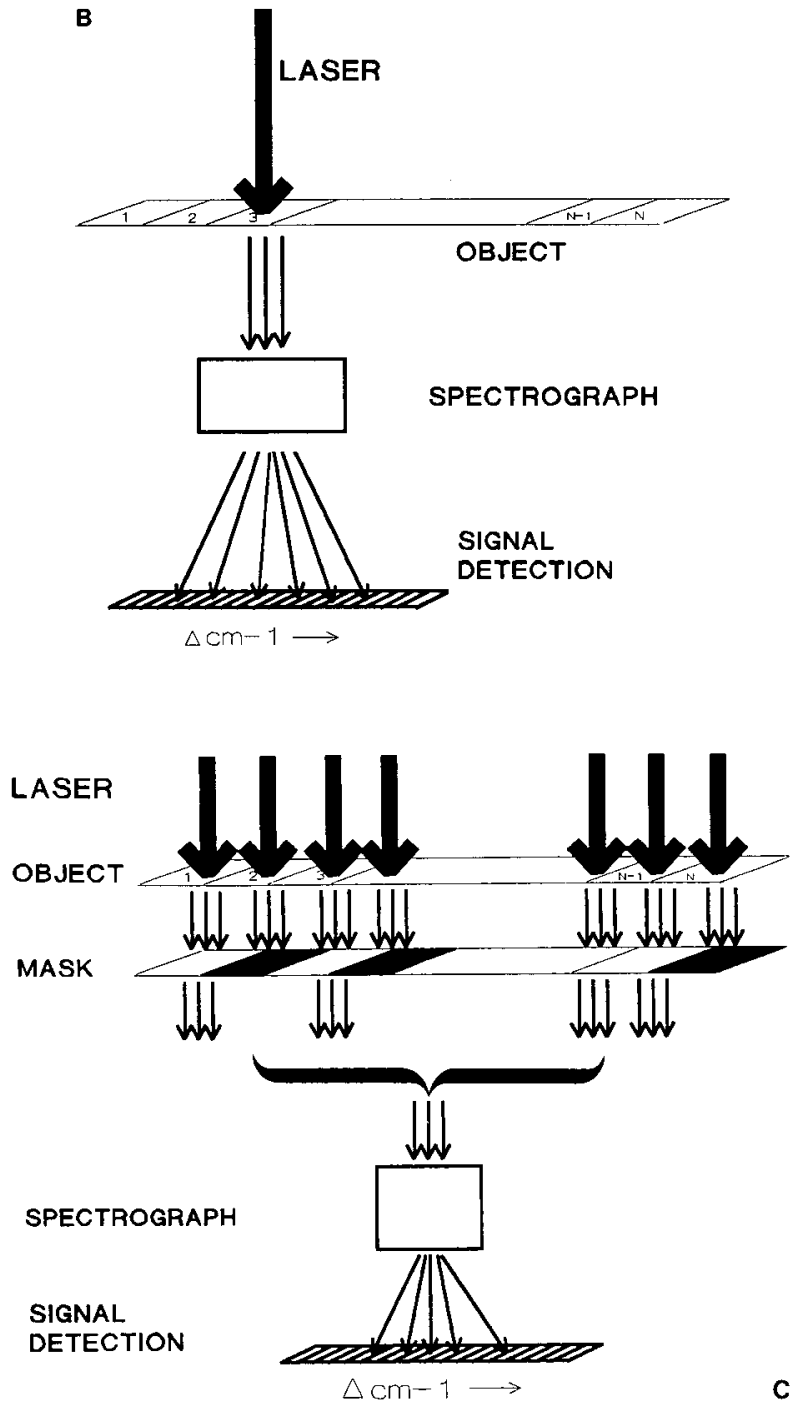

Fig. 1. Direct imaging and image reconstruction approaches to Raman microscopy: (A) Direct imaging. The object is globally illuminated with laser light. One wavenumber interval in the spectrum of the scattered light is selected, and an image of the object is formed on the basis of the intensity of the light scattered in this interval. (B) Image reconstruction after scanning the object pixel by pixel. In each measurement one pixel is illuminated with laser light. For every pixel a complete Raman spectrum is recorded. (C) Hadamard transform microscopy. The object is globally illuminated with laser light. A mask blocks the light scattered by about one half of the pixels. The spatial direction, encoded in this way, is compressed to a single point, of which the complete Raman spectrum is recorded. In order to reconstruct an image of the object consisting of $N$ pixels, $N$ measurements with linearly independent encodings are necessary.
The results will therefore directly apply to the case of 2D imaging.) There are three possible methods (see Fig. 1): (1) global illumination + signal detection at one wavenumber shift (Fig. 1A), repeated $M$ times; (2) pointillumination + complete Raman spectrum recording for all $N$ pixels separately (Fig. 1B); or (3) global illumination + complete Raman spectrum recording after (Hadamard) encoding of the spatial information at $N$ different settings of the encoding mask (Fig. 1C).

In each of the three methods, let every pixel be illuminated with the maximum allowable laser intensity (i.e., in method 2 the total laser power on the sample will be $N$ times lower than in the other two methods). In order to keep the derivation of the signal-to-noise ratio for the three methods simple and transparent it is assumed that a pixel contributes either a Raman signal $S$ or no Raman signal at all (see Fig. 2A). The fraction $a$ of pixels containing Raman signal is included in the calculations. Also included are a readout-noise $R$ and the fact that a background signal $B$-assumed to be the same for all pixels (e.g., due to buffer and substrate signal contributions in biological cell measurements) - to the Raman signal may be present, which has to be subtracted (Fig. 2B). It is furthermore assumed that when images are to be recorded at different wavenumber shifts (i.e., $M>1$ ), the Raman signal $S$ and the fraction of pixels containing Raman signal are the same for the different wavenumber shifts.

(1) Pixel Scanning. Suppose that a total period of time of $T_{0}$ seconds is available for the measurements (i.e., $T_{0} / N$ seconds for each pixel). We will define the Raman signal $S$ and background signal $B$ as the signals obtained from a pixel in $T_{0} / N$ seconds. In order to obtain a Raman image at a certain wavenumber shift $k$, the background signal, e.g., determined at a wavenumber shift $k+\Delta k$, has to be subtracted. The following expressions for signal and rms noise are then obtained for the image pixels:

For a pixel containing Raman signal:

signal:

$(S+B)-B=S$

rms noise:

$$
\sqrt{(S+B)+B+2 R^{2}}
$$

signal-to-noise ratio

$$
S / \sqrt{S+2 B+2 R^{2}} \text {. }
$$

The noise for a pixel not containing Raman signal becomes:

rms noise:

$$
\sqrt{2 B+2 R^{2}} \text {. }
$$

(2) Hadamard Transform. The available time $T_{0}$ is again divided over $N$ measurements (one at each setting of the Hadamard encoding mask. $S$ and $B$ are defined as under point 1 . It is assumed that on average the mask blocks the signal of half of the object pixels.

The following estimation of the signal-to-noise ratio for a pixel $i$ containing Raman signal can then be made: Of the total of $N$ measurements there will be $N / 2$ measurements with a signal contribution of pixel $i$. On average the total signal of a measurement containing a contribution from pixel $i$ will furthermore consist of signal (Raman and/or background) contributions of (N/2 - 1) other pixels.

If a fraction $a$ of all pixels contains Raman signal, then, again on average, a fraction $(a N-1) /(N-1)$ of these 
$(N / 2-1)$ other pixels will contribute Raman signal. Therefore the total Raman + background signal in each of the $N / 2$ measurements that include a signal contribution of pixel $i$ amounts to:

$$
\begin{aligned}
& S \times\left[1+(N / 2-1) \times \frac{(a N-1)}{(N-1)}\right]+B \times[1+(N / 2-1)](5) \\
& \text { contribution contribution contribu- contribution } \\
& \text { pixel } i \quad(N / 2-1) \text { tion }(N / 2-1) \\
& \text { other pixels pixel } i \text { other pixels }
\end{aligned}
$$

and the signal of all these $N / 2$ measurements added together gives:

$$
\begin{gathered}
N / 2 \times\left\{S \times\left[1+(N / 2-1) \times \frac{(a N-1)}{(N-1)}\right]\right. \\
+B \times[1+(N / 2-1)]\} .
\end{gathered}
$$

Now, it is clear that the result of the Hadamard backtransformation needed to reconstruct the signal of pixel $i$ is the subtraction of all signal of Eq. 5a that is not contributed by pixel $i$. Raman + background signal from pixel $i$ (at wavenumber shift $k$ ):

$$
\begin{gathered}
N / 2 \times\left\{S \times\left[1+(N / 2-1) \times \frac{(a N-1)}{(N-1)}\right]\right. \\
+B \times[1+(N / 2-1)]\} \\
(\text { term A) } \\
-N / 2 \times\left\{S \times(N / 2-1) \times \frac{(a N-1)}{(N-1)}+B \times(N / 2-1)\right\} \\
\quad(\text { term B) } \\
=N / 2 \times(S+B)
\end{gathered}
$$

Since the A-term and B-term in Eq. 6 are the results of independent measurements (namely, measurements with a contribution from pixel $i$ and measurements without a contribution of pixel $i$, respectively), the expression for the rms noise of pixel $i$ becomes:

rms noise:

$$
\begin{aligned}
& \sqrt{(\operatorname{term} \mathbf{A})+(\operatorname{term} \mathbf{B})+N \times R^{2}} \\
& =\left(N / 2 \times\left\{S \times\left[a N-\frac{(a N-1)}{(N-1)}\right]\right.\right. \\
& \left.+B \times(N-1)\}+N \times R^{2}\right)^{1 / 2} .
\end{aligned}
$$

The determination of the background signal contribution to pixel $i$ (at wavenumber shift $k+\Delta k$ ) proceeds along the same lines:

background signal pixel $i$ :

$$
\begin{aligned}
B & \times N / 2 \times[1+(N / 2-1)] \\
& -B \times N / 2 \times(N / 2-1)=B \times N / 2
\end{aligned}
$$

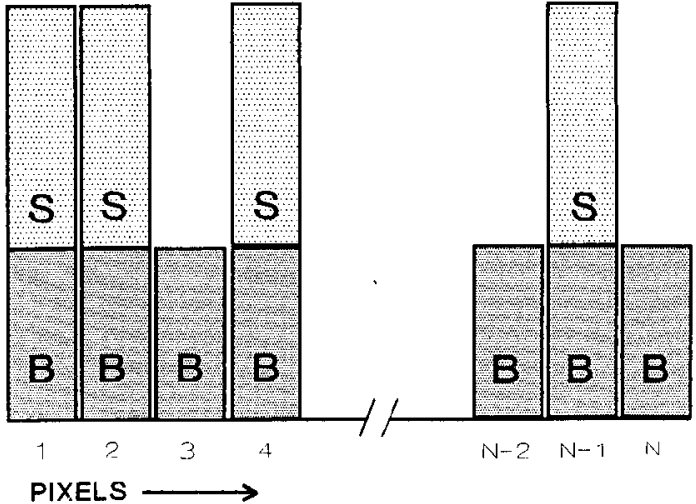

A

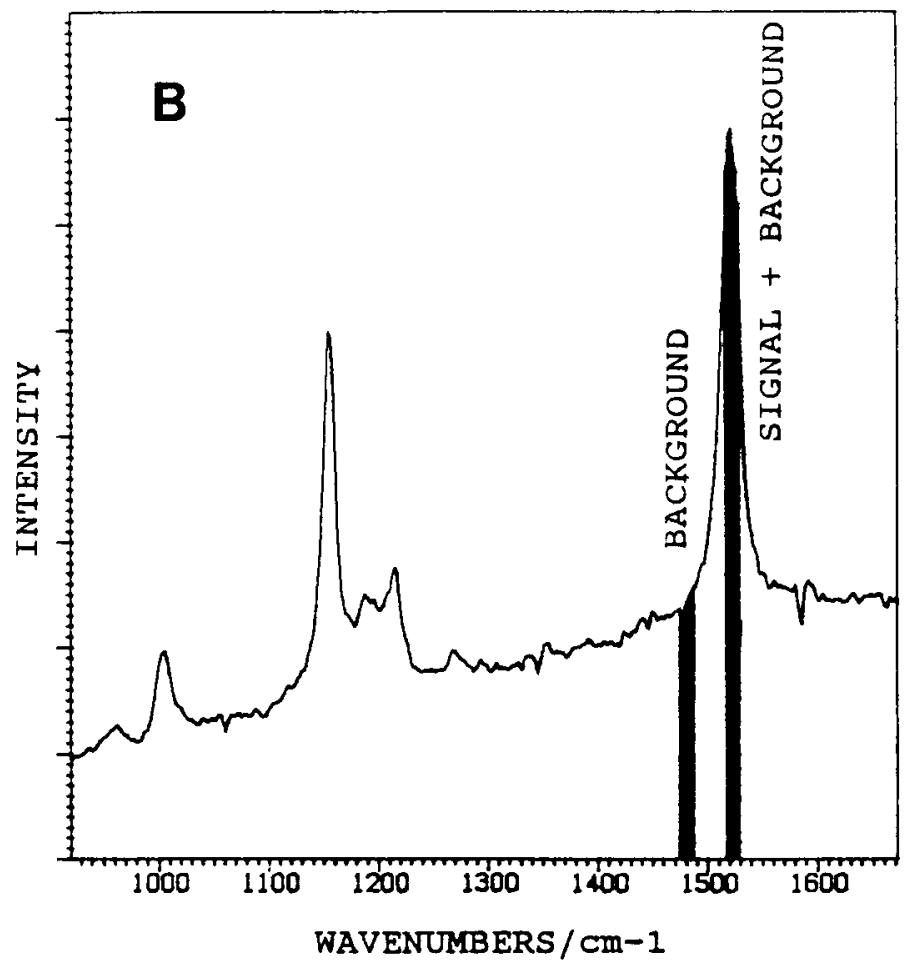

FIG. 2. Illustration of the model used to compare the performance in terms of signal-to-noise for different Raman imaging methods. (A) A 1D object is assumed, consisting of $N$ pixels. Each pixel has a background signal $B$ and a fraction $a$ of the pixels contains a Raman signal $S$. (B) The background signal contribution to the total signal is determined as shown in this carotenoid spectrum. $S+B$ is determined in the wavenumber interval covering the Raman line used for obtaining a Raman image; $B$ is determined in a wavenumber interval close to this Raman line. $S$ is found by simple pixel-by-pixel subtraction of the two images thus obtained.

rms noise:

$$
\sqrt{N / 2 \times(N-1) \times B+N \times R^{2}} \text {. }
$$

From Eqs. 6, 7, 8, and 9 then follow the expressions for the Raman signal obtained from pixel $i$ and the noise on this signal:

Raman signal pixel $i$ :

$$
\text { (6) }-(8)=S \times N / 2
$$

$$
\text { rms noise: } \quad \sqrt{(7)^{2}+(9)^{2}}=\left(N / 2 \times\left\{S \times\left[a N-\frac{(a N-1)}{(N-1)}\right]+2 B \times(N-1)\right\}+2 N \times R^{2}\right)^{1 / 2} .
$$


For $N \gg 1$, Eq. 11 becomes:

$$
\text { rms noise: } \sqrt{\left(N^{2} / 2\right) \times(a S+2 B)+2 N \times R^{2}} \text {. }
$$

Finally from Eqs. 10 and 12 follows the expression for the signal-to-noise ratio:

$$
S / \sqrt{2 a S+4 B+8 R^{2} / \bar{N}}
$$

(3) Direct Imaging. The Raman signal $S$ and background signal $B$ are defined as under points 1 and 2 above. In order to obtain a Raman image at a wavenumber shift $i$, two measurements $m 1$ and $m 2$ are necessary: $m 1$ at wavenumber shift $i$ and $m 2$ at $i+\Delta i$ in order to determine the background signal level. Subtraction of the second measurement from the first yields the desired Raman image. When $M$ images have to be made, for each image $T_{0} / M$ seconds are available. This leads to the following signal-to-noise ratio for each of the $M$ images:

For pixels containing Raman signal:

signal:

$$
\begin{aligned}
m 1-m 2 & =(S+B) \times N / 2 M-(B) \times N / 2 M \\
& =S \times N / 2 M
\end{aligned}
$$

rms noise:

$$
\sqrt{m 1+m 2+2 R^{2}}=\sqrt{(S+2 B) \times N / 2 M+2 R^{2}}
$$

signal-to-noise ratio:

$$
S \times \sqrt{N / 2 M} / \sqrt{S+2 B+2 R^{2} \times 2 M / N} .
$$

For pixels not containing Raman signal, only noise remains:

$$
\text { rms noise } \sqrt{(B \times N) / M+2 R^{2}} \text {. }
$$

Comparison of Eqs. 3, 13, and 16 for the signal-to-noise ratios of the different Raman imaging methods already gives a good insight into the performance characteristics of the different methods.

Under the condition that $R \ll S+2 B$, which is usually met when one is utilizing a cryogenically cooled CCD camera, the points discussed below become clear.

(1) Comparison of Direct Imaging and Pixel Scanning. The direct imaging method yields a signal-to-noise ratio higher than that for the pixel-scanning method by a factor $\sqrt{N / 2 M}$. This means that, until the number of different images $M$ that need to be recorded is bigger than the number of pixels in the scanned spatial dimension divided by two, the direct imaging method is to be preferred. In order to have a reasonably large field of view and good spatial resolution, $N$ will in general be on the order of 100 or larger. On the other hand, it is not easy to imagine many useful applications for which $M$ $>50$ (thus far there is no mention of such applications in the literature). Therefore in (most) practical situations, direct imaging will perform better than pixel-scanning.

(2) Comparison of Direct Imaging with Hadamard Transform Microscopy. This comparison is a little more complex. In the Hadamard transform method, the Raman signal shot-noise contribution to the total noise depends on $a$, the fraction of pixels containing Raman signal (see Eqs. 12 and 13). This is caused by the fact that the signal shot noise is equally distributed over all pixels (in fact expression 12 is valid for all pixels, whether or not they contain Raman signal). Therefore, in those cases where the background signal $B$ does not dominate the Raman signal $S$, this effect leads to an improvement in the signal-to-noise ratio, for pixels containing Raman signal.

In order to compare the performance of the direct imaging method (signal-to-noise ratio depends on $S, B$, $R, N$, and $M$ ) and the Hadamard transform method (signal-to-noise ratio depends on $S, B, R, N$, and $a$ ), calculations were made. The signal-to-noise ratio was calculated for both methods taking $N=100$ and $R=4(4$ electrons, equaling 4 detected photons) and for signal levels $S$ of $1,10,100$, and 1000 detected photons. The signal-to-background ratio $S / B$ was varied between 0.2 and 10 (stepsize 0.2 ). The number of images $M$ was varied between 1 and 40 (stepsize 1). The fraction of Raman signal containing pixels $a$ was varied between 0.02 and 1 (stepsize 0.02).

The results of these calculations are shown in Figs. 3 and 4 . Figures $3 \mathrm{~A}-3 \mathrm{H}$ show how the signal-to-noise ratio depends on $S / B$ and $M$ for direct imaging and $S / B$ and $a$ for the Hadamard method.

In Figs. $4 \mathrm{~A}-4 \mathrm{D}$ is shown in which part of the $(S / B, a$, $M)$-space the signal-to-noise ratio of the direct imaging method is higher than that obtained by the Hadamard transform method. It illustrates convincingly that only in a limited number of cases (small $a$, large $S / B$, and large $M$ ) does the Hadamard transform method outperform direct imaging. From the trend that, with decreasing Raman signal level $S$, the part of the $(S / B, a, M)$ space occupied by the Hadamard transform method grows, because the influence of the detector readoutnoise grows, it might appear that for very low signal levels this method will ultimately be the best choice. However, from $\mathrm{Fig}$. 3E it follows that, at these very low signal levels, signal-to-noise ratios $<\mathbf{1}$ are obtained, which are not of much practical use.

(3) Comparison of the Hadamard Transform and the Pixel-scanning Method. Comparison of Eqs. 3 and 13 shows that the classical multiplexing advantage of the Hadamard transform method exists only if the readoutnoise $R$ is the dominating noise term. As was illustrated above, this situation occurs only under conditions that are not of much practical use, when a liquid nitrogencooled CCD camera is used for signal detection. The second "advantage" of the Hadamard transform method is that the Raman signal shot noise is equally divided over all pixels. This advantage will be present especially then when $a$ is small and $S / B$ large. In that case, the Raman signal shot noise term will dominate in Eq. 3, while in Eq. 13 it may still be small. However, the noise level for the pixels that do not contain Raman signal is in the Hadamard transform method partly determined by the signal $S$ of the pixels that do contain Raman signal. This factor negatively influences the dynamic range of the method, because this will make it harder to detect the signal of pixels with a small Raman signal in the presence of pixels with a strong Raman signal. It therefore depends on the precise application whether or not this second "advantage" is really an advantage.

From these considerations it follows that, in terms of signal-to-noise ratio (or measuring time needed to obtain the desired images with a certain signal-to-noise ratio), 

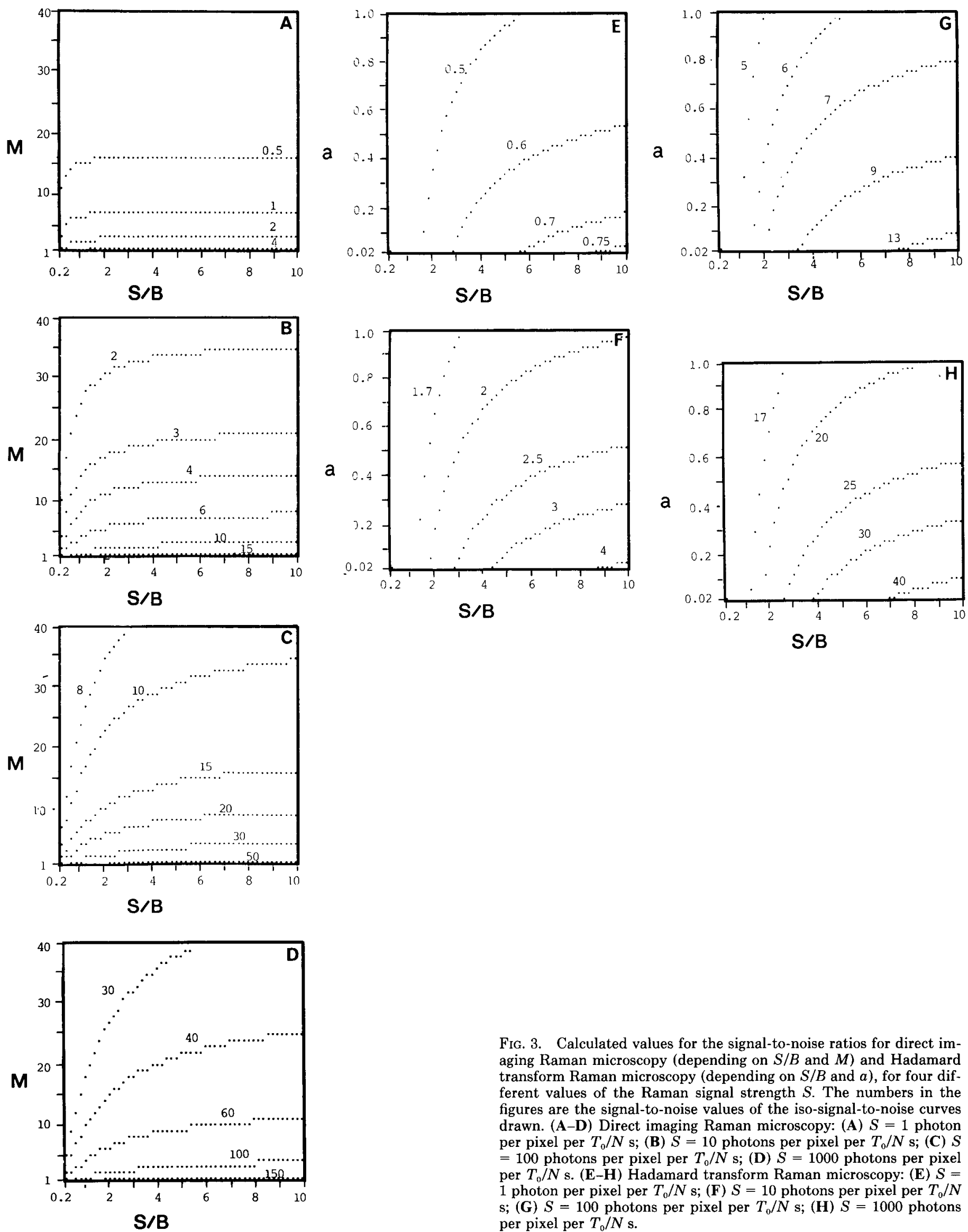

FIG. 3. Calculated values for the signal-to-noise ratios for direct imaging Raman microscopy (depending on $S / B$ and $M$ ) and Hadamard transform Raman microscopy (depending on $S / B$ and $a$ ), for four different values of the Raman signal strength $S$. The numbers in the figures are the signal-to-noise values of the iso-signal-to-noise curves drawn. (A-D) Direct imaging Raman microscopy: (A) $S=1$ photon per pixel per $T_{0} / N \mathrm{~s}$; (B) $S=10$ photons per pixel per $T_{0} / N$ s; (C) $S$ $=100$ photons per pixel per $T_{0} / N \mathrm{~s}$; (D) $S=1000$ photons per pixel per $T_{0} / N$ s. (E-H) Hadamard transform Raman microscopy: (E) $S=$ 1 photon per pixel per $T_{0} / N \mathrm{~s}$; (F) $S=10$ photons per pixel per $T_{0} / N$ s; (G) $S=100$ photons per pixel per $T_{0} / N \mathrm{~s}$; (H) $S=1000$ photons per pixel per $T_{0} / N$ s. 

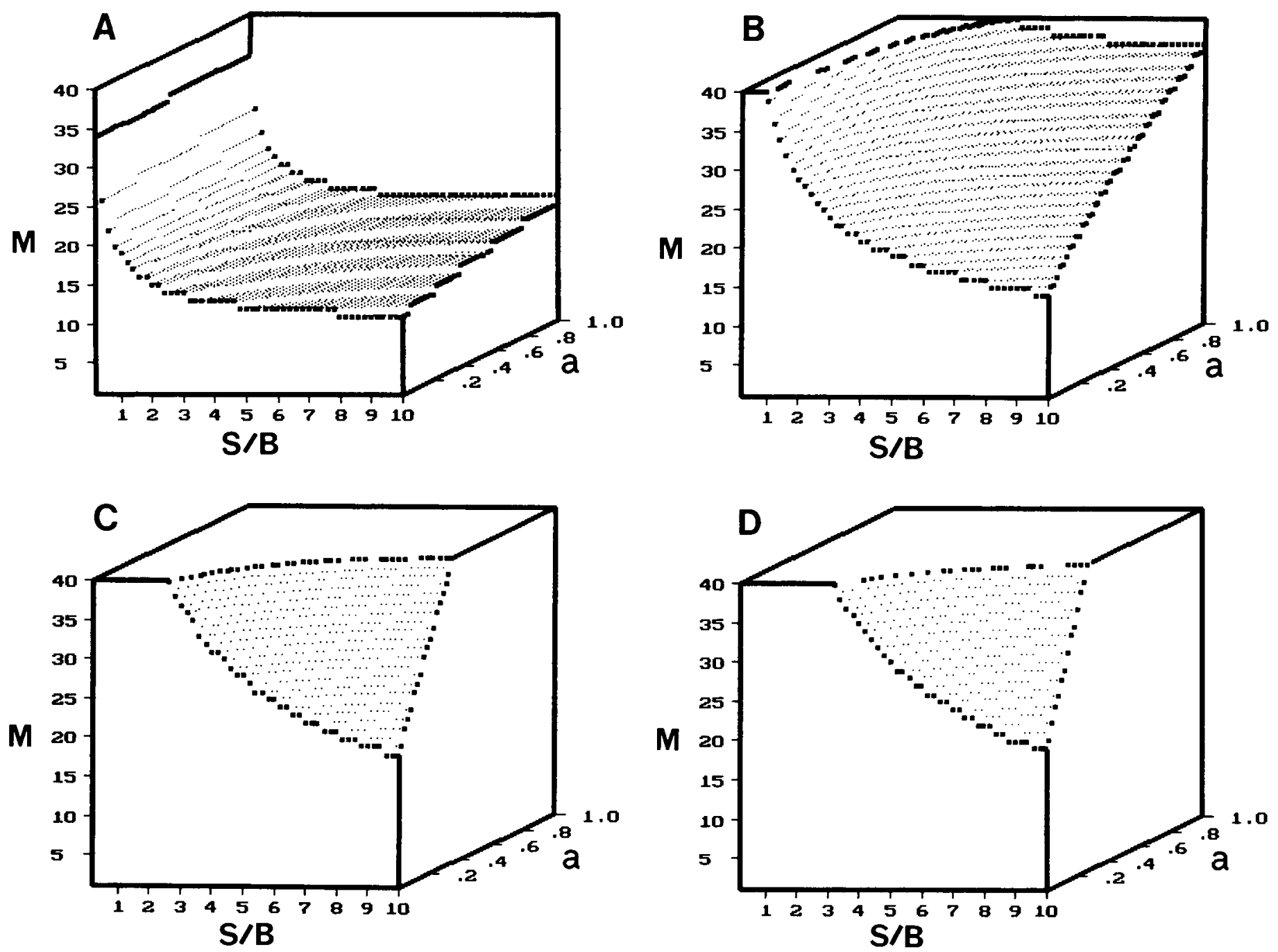

FIG. 4. Graphical presentation of a calculated comparison of the signal-to-noise ratios obtained by direct imaging Raman microscopy and Hadamard transform Raman microscopy for four different values of the Raman signal strength $S$. The enclosed part of the $(S / B$, $a, M)$-cube denotes the $(S / B, a, M)$-space in which the direct imaging method performs better than the Hadamard transform method. It can be seen that, with increasing signal strength $S$, the part of the $(S / B, a, M)$-space where the Hadamard transform method performs better (i.e., the excluded part of the cube) becomes smaller. (A) $S=1$ photon per pixel per $T_{0} / N \mathrm{~s} ;$ (B) $S=10$ photons per pixel per $T_{0} / N \mathrm{~s}$; (C) $S=100$ photons per pixel per $T_{0} / N \mathrm{~s}$; (D) $S=1000$ photons per pixel per $T_{0} / N \mathrm{~s}$.

direct imaging methods will in almost all practical cases outperform image reconstruction methods. Comparing the two image reconstruction methods, it is furthermore evident that, when one is using a slow-scan CCD camera for signal detection, the Hadamard transform method does not offer a general advantage over the line-scanning method. One exception may be the experiment in Ref. 11 where a carbon electrode sample needed $15 \mathrm{~min}$ of thermal equilibration in the laser light prior to the measurements. With the use of line-scanning for each new line, this thermal equilibration would take $15 \mathrm{~min}$. This observation, however, points out another advantage of global illumination rather than a specific advantage of Hadamard transform imaging.

Changing the parameters of the model used for the calculations (e.g., $B$ not equal for all pixels, but only present for Raman signal containing pixels, larger $R$ or smaller $N$ ), which may be more appropriate for modeling some practical situations, changes the details of the re- sults. But the general picture-that in most cases direct imaging is to be preferred-will remain the same.

An advantage of the image reconstruction methods that has not yet been mentioned is that the decision of precisely how to integrate the intensity of a Raman line and how to determine the amount of background signal that has to be subtracted can be made after the measurements have been collected, because for every pixel a complete Raman spectrum is recorded. This approach is not possible in the direct imaging methods.

On the other hand, the direct imaging methods have the advantage that the signal integration time can be adjusted for each individual Raman image, depending on how intense the Raman signal is at a certain wavenumber shift. In the image reconstruction methods the total measuring time has to be adjusted in such a way that, even at the wavenumber shift at which the Raman signal is lowest, a good image is obtained.

Additional disadvantages of the Hadamard transform 


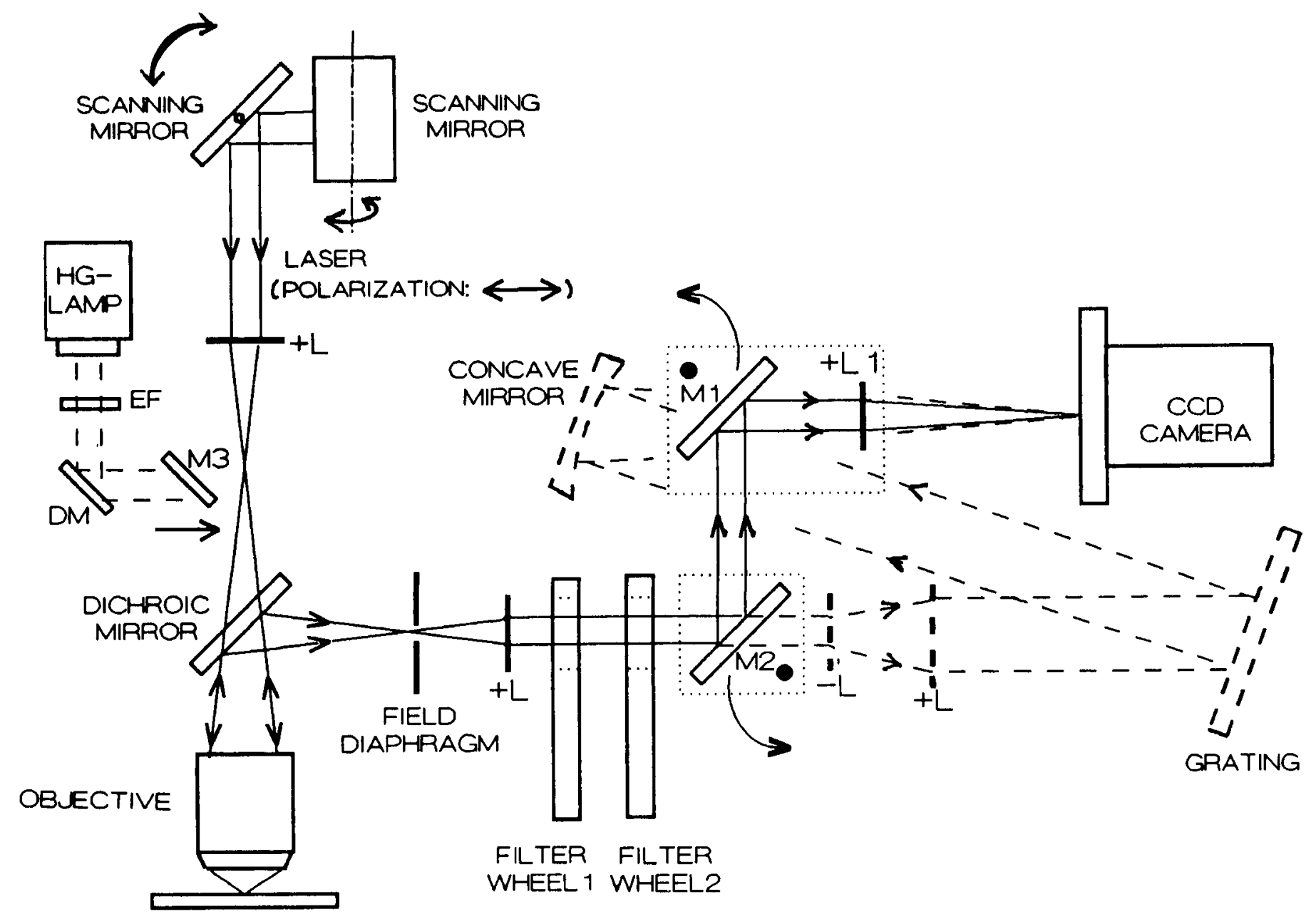

FIg. 5. Schematic of the direct imaging Raman microscope described in this communication (also see text). Filter wheel 1 contains Raman holographic edge filters and color-glass cutoff filters, filter wheel 2 contains two dielectric narrow-band transmission filters and a number of empty positions. M1 and $+\mathbf{L 1}$, and M2, are fixed on rotatable mounts (hatched lines), which can be swung out of the path of light, in order to switch from imaging to spectroscopy mode. In that case filter wheel $\mathbf{2}$ is rotated to an empty position. M3 can be positioned in the path of the laser beam, enabling fluorescence excitation. A cutoff color-glass filter in filter wheel 1 is then used for suppression of the exciting light from the Hglamp and filter wheel 2 is in an empty position. Abbreviations: L: lens, M: mirror, EF: fluorescence excitation filter, DM: dichroic mirror.

method are that mask defects and mask positioning errors can arise, which degrade image quality. ${ }^{10,15}$

Of course, the power distribution advantage of direct imaging microscopes is utilized only if samples are vulnerable to laser-light-induced damage, when one is linefocusing all available laser power onto the sample. However, in biological applications (e.g., the imaging of cells and chromosomes) which are the focus of our attention, this will usually be the case.

Because of the considerations given above we have initiated the development of sensitive direct imaging $\mathrm{Ra}$ man microscopes.

\section{EXPERIMENTAL}

A direct imaging Raman microscope was built, with high spatial and spectral resolution and efficient signal detection (Fig. 5). The key elements of the design are a narrow-band dielectric transmission filter (Omega Optical, Inc., Brattleboro, VT; FWHM of transmission band $=0.5 \mathrm{~nm}$ ) and an argon-ion laser pumped tunable dye laser (Spectra-Physics, Model 375 B). The filter is used to select one wavelength interval from the spectrum of the light that is scattered by the object (Fig. 6). A Raman image can be recorded at the desired Raman shift by tuning the wavelength of the dye laser in such a way that the wavelength of the Raman scattered light of the desired wavenumber coincides with the wavelength of the transmission band of the narrow-band dielectric filter.

The dye laser is operated with DCM (4-dicyanomethylene-2-methyl-6-[(p-dimethylamino)styryl]-4H-pyran). The laser wavelength is tuned between 630 and 690 $\mathrm{nm}$ by means of a computer (PC-XT)-controlled stepper motor (Maerzhauser EK-32), attached to the birefringent filter of the dye laser. Two dielectric narrow-band transmission filters $(711 \mathrm{~nm}$ and $735 \mathrm{~nm}$ ) have been used in this work, both with a peak transmittance of $60 \%$. Transmittance outside the pass band is $<10^{-3}$ (from UV to $>1000 \mathrm{~nm}$ ). Their transmission bandwidth of $0.5 \mathrm{~nm}$ (FWHM) provides a spectral resolution of $10 \mathrm{~cm}^{-1}$.

The sample is globally illuminated. In order to obtain an homogeneous illumination, the object is rapidly 


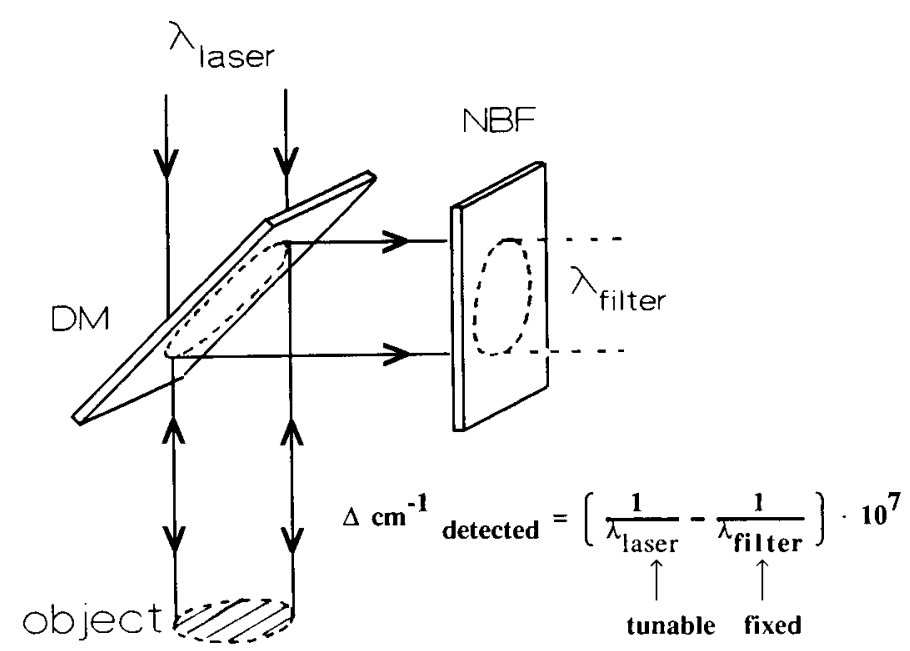

FIG. 6. Illustration of the principle of selecting a wavenumber interval for Raman imaging. The wavelength of the laser light is tuned to make the wavelength of the desired Raman band coincide with the transmission band of the dielectric narrow-bandpass filter (NBF). DM = dichroic mirror. $\lambda$ in $\mathrm{nm}$.

scanned with a focused laser beam. To this end, two scanning mirrors (Philips, Type 4822380 20118) are placed near the conjugate plane of the entrance pupil of the objective, so that during the scan the objective aperture is completely filled without loss of laser power on the sample (method described in Ref. 16). Both mirrors are driven with triangular waveforms, one at a frequency of $30 \mathrm{~Hz}$, the other at $0.5 \mathrm{~Hz}$. Since there is no phase relation between the movements of the two mirrors, because independent drivers are used, an effectively homogeneous illumination is obtained after a few complete scans of the object, i.e., in a period of time that is short compared with the duration of the measurements. Unless nonlinear effects play a role, the same laser power can be used that is used when the sample is globally illuminated with a defocused laser beam (which, however, gives rise to a Gaussian instead of a homogeneous intensity distribution in the object plane).

A dielectric dichroic mirror (produced in the thin-film laboratory of our department) is used to optically couple the microscope with the detection arm of the setup. Its filter characteristics (Fig. 7) are such that, in the wavelength interval covered by the dye laser, it has a high transmittance (i.e. $\sim 95 \%$ in the wavelength interval 620 to $690 \mathrm{~nm}$; the laser light is $p$-polarized). Above $690 \mathrm{~nm}$, reflection (for $p$-polarization) steeply rises to $>98 \%$.

Laser light suppression in the detection arm is accomplished by a set of Raman holographic edge filters (center wavelengths: $660,670,680$, and $690 \mathrm{~nm}$, obtained from Physical Optics Corp., Torrance, CA, and Kaiser Optical Systems, Inc., Ann Arbor, MI) held in a filter wheel, so that, for each laser wavelength, the right filter can be quickly installed. For laser wavelengths $<660 \mathrm{~nm}$, an anti-reflection-coated color-glass cutoff filter (RG 695, Schott, Tiel, The Netherlands) is used. Total laser light suppression by dichroic mirror, holographic edge filter, and dielectric narrow-band transmission filter is $>10^{9}$ at any laser wavelength, between 630 and $690 \mathrm{~nm}$.

The signal detection efficiency of the setup is such that, of all the photons scattered within the solid angle of

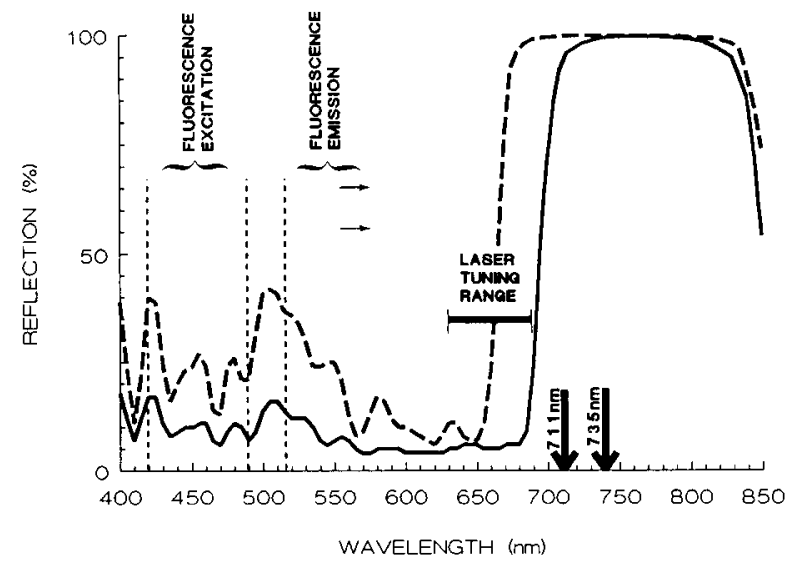

Fig. 7. Transmission spectrum of the dichroic mirror used to optically couple the microscope and the spectrometer: $(-) p$-polarization; $(\cdots \cdots) s$-polarization. Indicated in the figure are the positions of the transmission band of the dielectric narrow-bandpass filters used in this work (711 and $735 \mathrm{~nm}$ ), the tuning range of the dye laser (laser light p-polarized), and the fluorescence excitation and emission bands.

collection of the microscope objective and at the transmission wavelength of the dielectric narrow-bandpass filter, $\sim 15 \%$ will be detected by the slow-scan liquid nitrogen CCD camera (Model AT1 equipped with an English Electric Valve P8603 CCD chip, Wright Instruments Ltd., Enfield, U.K.). This figure includes all reflection and transmission losses in the setup as well as the $\sim 40 \%$ quantum efficiency of the (front illuminated) CCD chip.

In most applications a $\times 63$ microscope objective (Zeiss Plan Neofluar, numerical aperture 1.2, water immersion) is used. At the signal detection wavelengths (711 or 735 $\mathrm{nm}$ ) the diffraction limit of the spatial resolution is, therefore, according to Abbe's rule $\sim 0.35 \mu \mathrm{m}$. The total magnification of the image of the CCD camera is $\times 190$. The pixel size of the CCD chip is $22 \times 22 \mu \mathrm{m}^{2}$. Therefore, the object is sampled with $8-9$ pixels $/ \mu \mathrm{m}$, preventing deterioration of spatial resolution.

The in-base illumination system of the microscope frame is used for obtaining bright field microscopic images.

The reflection peaks in the dichroic mirror transmission spectrum at lower wavelengths (Fig. 5) enable incorporation of an epi-illumination fluorescence microscopy option (Fig. 5). When the setup is used in fluorescence mode, mirror M3 is inserted in the path of the laser beam directing light from the $\mathrm{Hg}$-lamp towards the sample. A cutoff color-glass filter (OG 515, Schott, Tiel, The Netherlands) in filter wheel 1 is used to suppress fluorescence excitation light from the Hg-lamp. Filter wheel 2, containing the dielectric narrow-bandpass filters, is turned to an empty position. In this work the epi-illuminescence arm and Hg-lamp of a Nikon Optiphot fluorescence microscope were used together with a 420-490 $\mathrm{nm}$ excitation filter and a dichroic mirror (DM 510) for blue fluorescence excitation.

The Raman/fluorescence/bright field imaging setup is incorporated in a Raman microspectrometer, described previously (Refs. 1 and 17), enabling easy switching between imaging mode and multichannel microspectroscopy mode (Fig. 5). 


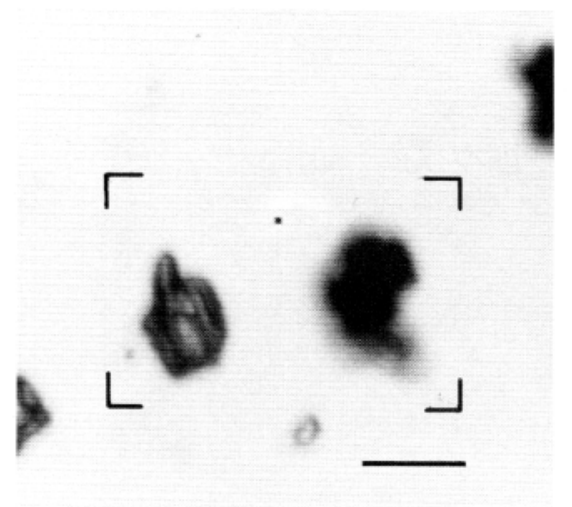

A
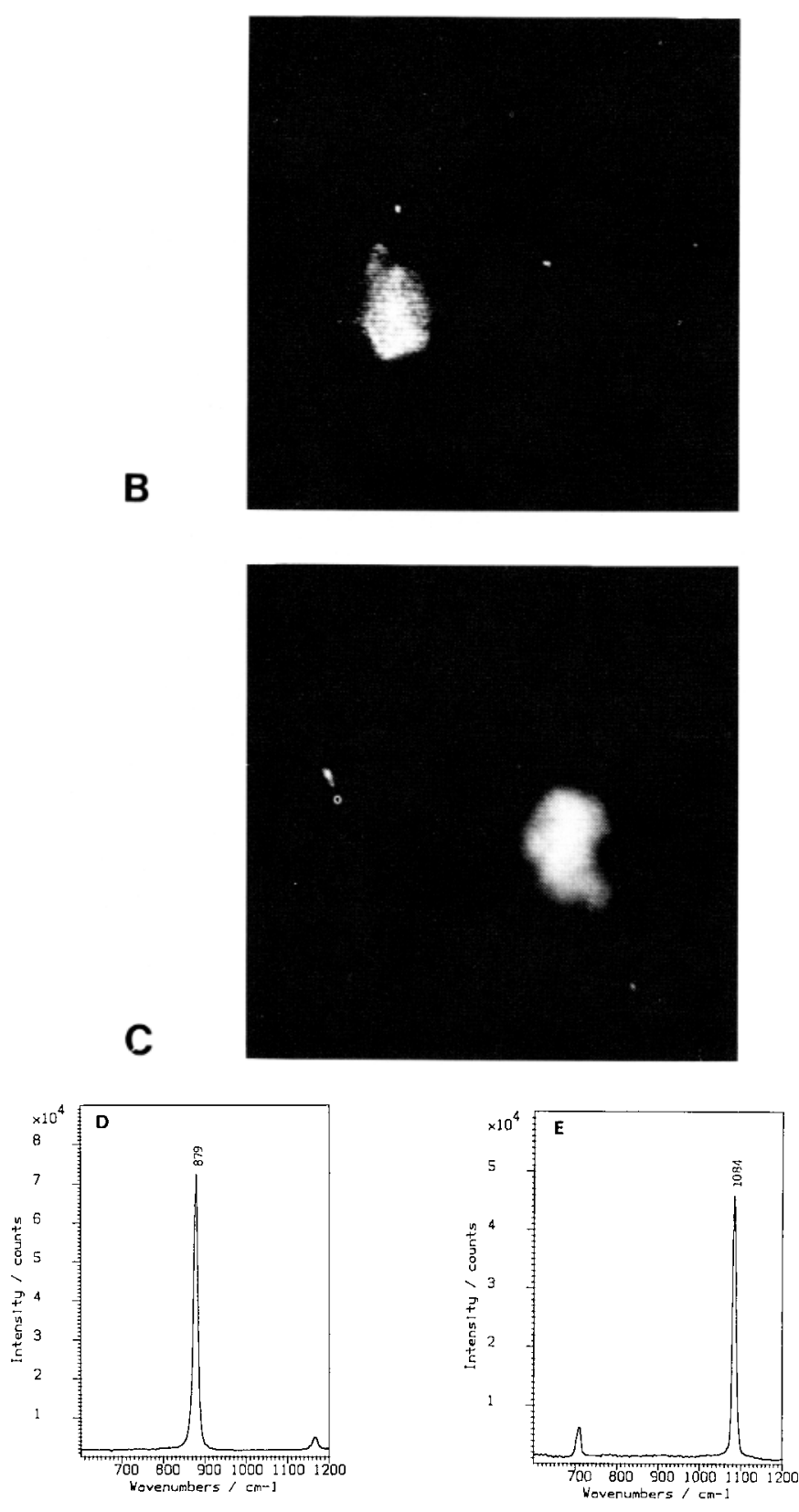

FIG. 8. (A) Bright field image of boric acid (left) and calcite (right) crystals. Bar denotes $20 \mu \mathrm{m}$. The part of the sample that was illuminated with laser light is indicated. (B) Raman image showing only boric acid crystal (image recorded at $879 \mathrm{~cm}^{-1}$ minus background image
The wavenumber calibration of the Raman microscope is carried out with the setup in spectrometer mode. The in-base illumination system of the microscope is switched on to produce a white light spectrum. With the dielectric narrow-bandpass filter in place, the spectrum recorded by the CCD camera is a single narrow line (FWHM 10 $\mathrm{cm}^{-1}$ ), indicating the position on the camera of the wavelength transmitted by the filter. The number $N_{\max }$ of the detector channel with highest signal intensity is noted. Then the dielectric narrow-band filter is removed, and a Raman spectrum of a sample of indene recorded. The position of the stepper motor, driving the birefringent filter of the dye laser, is adjusted until one of the indene Raman lines coincides with channel $N_{m a x}$. The stepper motor position with the corresponding Raman wavenumber shift is then stored in the computer memory. This procedure is repeated for at least five lines in the spectral interval of interest. After that, a third-order polynomial is fitted through these points, with a least-squares method applied.

After the calibration procedure, the setup is returned to the imaging mode. The desired wavenumber shift of the Raman image can be fed into the computer, after which the stepper motor tunes the birefringent filter of the dye laser to the correct position. The dye laser wavelength can be tuned in steps of $0.6 \mathrm{~cm}^{-1}$ and with a reproducibility of $\sim 2 \mathrm{~cm}^{-1}$.

With the use of DCM, the dye laser can be tuned over a relatively wide wavelength interval. The maximum of the DCM efficiency curve lies at about $655 \mathrm{~nm}$, while still $30 \%$ of the maximum efficiency is obtained at 630 and $690 \mathrm{~nm}$. Tuning the laser between 630 and $690 \mathrm{~nm}$ implies tuning of the Raman microscope over a wavenumber range of about $1350 \mathrm{~cm}^{-1}$. This range can be extended by enabling signal detection at more than one wavelength, with the use of a number of dielectric narrow-bandpass filters with different transmission bands (e.g., one filter with a center wavelength of $711 \mathrm{~nm}$ and one of $785 \mathrm{~nm}$ would make it possible to obtain Raman images at any wavenumber between 450 and $3100 \mathrm{~cm}^{-1}$ ).

\section{RESULTS AND DISCUSSION}

Batchelder et al. ${ }^{5}$ were the first to employ dielectric narrow-band filters in a Raman microscope. Their design is based on angle tuning of the dielectric filter in order to select a particular wavenumber shift for imaging. Our design has several advantages, however, because it avoids a number of anomalous effects encountered when a dielectric bandpass filter is used under conditions of nonnormal light incidence.

The filter transmission wavelength $\lambda(\vartheta)$ depends on the angle $\vartheta$ the incident light makes with the normal of the filter surface according to: ${ }^{5}$

recorded at $\left.850 \mathrm{~cm}^{-1}\right)$. Conditions for image measurements: laser power on sample: $12 \mathrm{~mW}$ (laser intensity: $\sim 300 \mathrm{~W} / \mathrm{cm}^{2}$ ); signal integration time: $600 \mathrm{~s} ; \times 10$ Spindler and Hoyer microscope objective (numerical aperture: 0.25). (C) Raman image showing only calcite crystal (1084 $\mathrm{cm}^{-1}$ image minus $1060 \mathrm{~cm}^{-1}$ background image). Measuring conditions: see B. (D) Raman spectrum of boric acid. (E) Raman spectrum of calcite. 


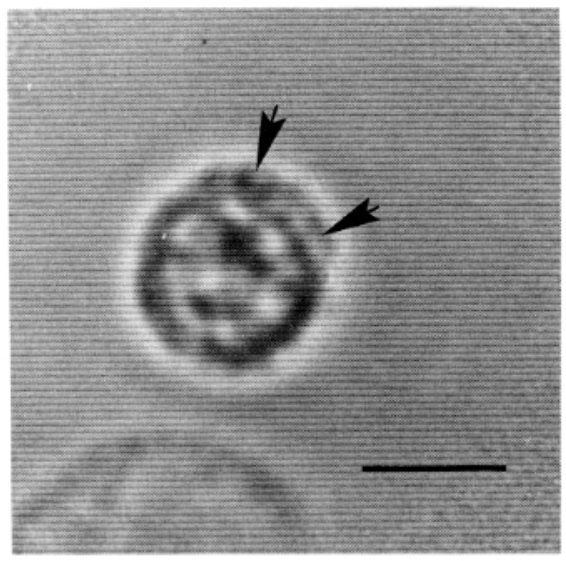

A

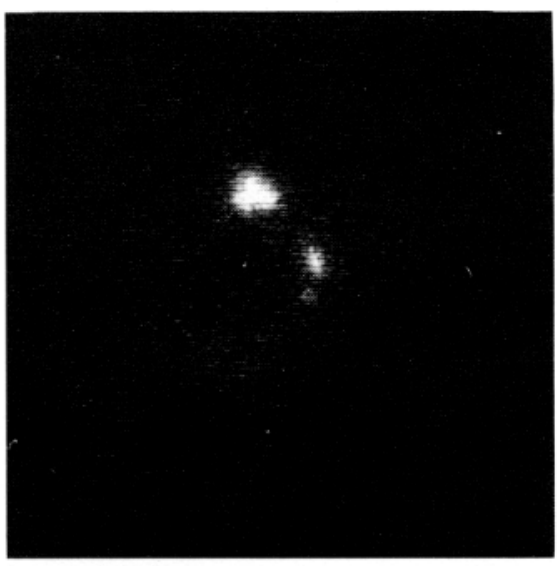

B

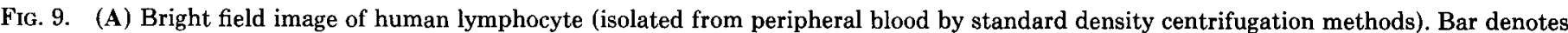

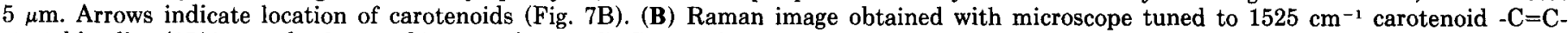

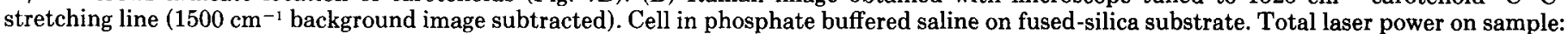

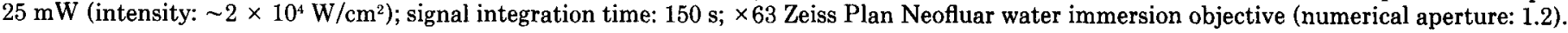

$$
\lambda(\vartheta)=\lambda \times \frac{\sqrt{\left(n^{2}-\sin ^{2} \vartheta\right)}}{n}
$$

( $n$ : effective index of refraction).

First, light coming from different parts of the object passes the dielectric narrow-bandpass filter under slightly different angles $\vartheta$ (vignetting). Therefore different parts of the object are imaged at different wavenumber shifts, leading to a spectrally inhomogeneous image. This effect is much stronger when the filter is used with non-normal light incidence, than under conditions of normal light incidence. In our setup, with the use of a $\times 63$ microscope objective, spectral inhomogeneity for a $50-\mu \mathrm{m}$ field of view is $<1 \mathrm{~cm}^{-1}$, which in principle enables one to obtain images with a spectral resolution of a few wavenumbers. With the use of, instead, a filter tuned $10^{\circ}$ away from normal incidence, spectral inhomogeneity would be $>20$ $\mathrm{cm}^{-1}$. This observation implies that, in the angle tuning method, either the field of view has to be kept very small or spectral resolution (determined by FWHM of the filter transmission band) has to be sacrificed.

Second, for $\vartheta>0$, the transmission bands for $p$ - and $s$-polarized light diverge. ${ }^{18}$ This means that different wavenumber intervals are transmitted for $p$ - and $s$-polarized Raman scattered light. With a polarizer one of the two needs to be blocked, thereby reducing signal detection efficiency. This loss of signal is avoided in our setup.

Signal detection efficiency and spectral resolution in the filter angle tuning method are furthermore degraded by the fact that, for dielectric narrow-band transmission filters, peak transmission usually decreases and transmission bandwidth increases with increasing $\vartheta$.

The Raman microscope, described by Treado et al., ${ }^{6}$ which is based on filtering Raman scattered light by an acousto-optical tunable filter, also has the disadvantage that only one polarization component of the scattered light can be used and that spectral resolution is low $(\sim 50$ $\mathrm{cm}^{-1}$ ).

In Figs. 8-10 a number of examples are given that illustrate the possibilities of our Raman microscope. Figure $8 \mathrm{~A}$ is a bright field image of two crystals of boric acid (left) and calcite (right). Figures $8 \mathrm{D}$ and $8 \mathrm{E}$ show the Raman spectra obtained from these crystals. After these spectra were recorded, the setup was switched to the imaging mode. Figure $8 \mathrm{~B}$ is the result of a measurement at $879 \mathrm{~cm}^{-1}$ (boric acid) from which a background image recorded at $850 \mathrm{~cm}^{-1}$ was subtracted on a pixel-by-pixel basis. Only the boric acid crystal is visible. Figure $8 \mathrm{C}$, showing the calcite crystal, was obtained after the microscope was tuned to $1084 \mathrm{~cm}^{-1}$ (background image recorded at $1060 \mathrm{~cm}^{-1}$ subtracted).

Figure $9 \mathrm{~A}$ is a bright field image of a human lymphocyte. Figure $9 \mathrm{~B}$ is a Raman image, showing the subcellular distribution of carotenoids in this cell. [It is the result of a measurement at $1525 \mathrm{~cm}^{-1}$ (carotenoid $-\mathrm{C}=\mathrm{C}$ in-phase stretching vibration) from which a background image obtained at $1500 \mathrm{~cm}^{-1}$ was subtracted.] It should be noted that the carotenoid signal was not resonantly enhanced; the laser wavelength was $660 \mathrm{~nm}$. Details about the subcellular distribution of carotenoids in human lymphocyte subpopulations can be found in Ref. 3 .

Figures 10A-10C are images of fluorescently labeled polystyrene beads (Polysciences fluoresbryte spheres, Warrington, PA). They illustrate the possibility of combining bright field, Raman, and fluorescence microscopy, provided that laser excitation for the Raman experiments occurs sufficiently far away from the absorption band of the fluorescent label. This will be a valuable option in many biological applications.

\section{CONCLUSIONS}

This paper and a number of recent papers by others have shown that Raman microscopy is a feasible chemical imaging technique. Because Raman signal intensity levels are usually very low, efficient signal detection is of utmost importance. Further improvements in the signal detection efficiency of our instrument will have to come from optimization of the dielectric narrow-bandpass filters (width and height of the transmission band) and 

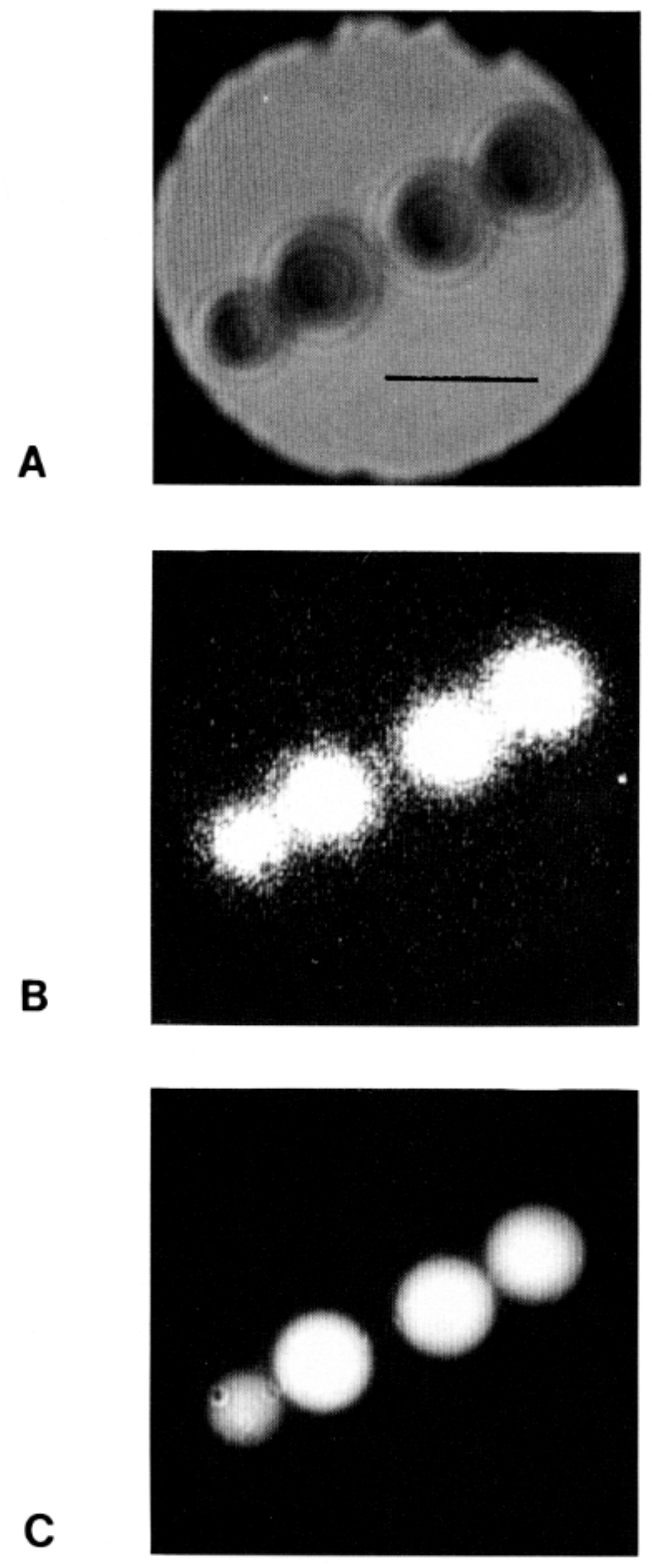

FIG. 10. (A) Bright field image of polystyrene spheres labelled with a fluorescent dye. Bar denotes $5 \mu \mathrm{m}$. [The image is perturbed due to diffraction and refraction of light by the spheres; the round dark edge of the image is due to the field diaphragm (see Fig. 3).] (B) Raman image obtained with microscope tuned to the $998-\mathrm{cm}^{-1}$ polystyrene line $\left(970-\mathrm{cm}^{-1}\right.$ background image subtracted). Laser power on sample: $15 \mathrm{~mW}$; (laser intensity: $\sim 8 \mathrm{KW} / \mathrm{cm}^{2}$ ); signal integration time: $150 \mathrm{~s}$; microscope objective: see legend of Fig. 7. (C) Fluorescence image obtained with blue excitation $(420-490 \mathrm{~nm})$ and emission detection $>515$ $\mathrm{nm}$ [two OG 515 color glass cutoff filters (thickness: $3 \mathrm{~mm}$ ) were used to block Hg-lamp excitation light].

from replacement of the front-illuminated CCD chip presently used (quantum efficiency at $700 \mathrm{~nm} \sim 40 \%$ ) by a thinned back-illuminated chip (quantum efficiency $\sim 80 \%$ ). In cases where it is not absolutely necessary to work at diffraction-limited spatial resolution, one can bin camera pixels in order to increase the number of detected photons per image pixel (presently 9 pixels $/ \mu \mathrm{m}$ are used).
As in the case of Raman microspectroscopy, ${ }^{1,17}$ signalto-noise ratios can also be improved through the suppression of unwanted signal contributions (i.e., background signal) from the environment of thin and/or weakly scattering objects by adopting a confocal detection scheme. Confocal detection has already been implemented in the (image reconstruction) instrument of Barbillat et al. ${ }^{8}$

Disadvantages of filter-based Raman microscopes (including the one described here) are that the bandwidth of the filter cannot be adjusted to create optimum detection conditions for each application and that access to the low-wavenumber region is difficult. Therefore a direct imaging confocal Raman microscope, which employs a grating monochromator for the selection of the wavenumber shift and interval, appears to be the ultimate solution for Raman microscopy. We expect that with such an instrument it will be possible to make images of, e.g., the DNA and protein distribution in cells and chromosomes.

\section{ACKNOWLEDGMENT}

The authors wish to thank Tom Bakker Schut for his help in writing a computer code for presentation of the results of signal-to-noise calculations.

1. G. J. Puppels, F. F. M. de Mul, C. Otto, J. Greve, M. RobertNicoud, D. J. Arndt-Jovin, and T. M. Jovin, Nature 347, 301 (1990).

2. I. Siebinga, G. F. J. M. Vrensen, F. F. M. de Mul, and J. Greve, Exp. Eye Res. 53, 233 (1991).

3. G. J. Puppels, H. S. P. Garritsen, J. A. Kummer, and J. Greve, Cytometry 14(3), 251 (1993).

4. M. Delhaye and P. Dhamelincourt, J. Raman Spectrosc. 3, 33 (1975).

5. D. N. Batchelder, C. Cheng, W. Mueller, and B. J. E. Smith, Makromol. Chem., Macromol. Symp. 46, 171 (1991).

6. P. J. Treado, I. W. Levin, and E. N. Lewis, Appl. Spectrosc. 46, 1211 (1992).

7. M. Bowden, D. J. Gardiner, G. Rice, and D. L. Gerrard, J. Raman Spectrosc. 21, 37 (1990).

8. J. Barbillat, E. da Silva, M. Delhaye, P. Dhamelincourt, M. Manfait, and B. Roussel, in Proceedings of the 13th International Conference on Raman Spectroscopy, (John Wiley \& Sons, Chichester, 1992), pp. 1074-1075.

9. P. J. Treado and M. D. Morris, Appl. Spectrosc. 44, 1 (1990).

10. P. J. Treado, A. Govil, M. D. Morris, K. D. Sternitzke, and R. L. McCreery, Appl. Spectrosc. 44, 1270 (1990).

11. P. J. Treado and M. D. Morris, Spectrochim. Acta Rev. 13, 355 (1990).

12. K.-L. K. Liu, L.-H. Chen, R.-S. Sheng, and M. D. Morris, Appl. Spectrosc. 45, 1717 (1991).

13. R. J. Bell, Introductory Fourier Transform Spectroscopy (Academic Press, New York, 1972).

14. D. B. Chase, in Analytical Raman Spectroscopy, J. G. Grasselli and B. J. Bulkin, Eds. (John Wiley \& Sons, New York, 1991), pp. $21-43$.

15. M. H. Tai, M. Harwitt, and M. J. A. Sloane, Appl. Opt. 14, 2678 (1975).

16. T. Wilson and C. Sheppard, Theory and Practice of Scanning Optical Microscopy (Academic Press, London, 1984), pp. 173-175.

17. G. J. Puppels, W. Colier, J. H. F. Olminkhof, C. Otto, F. F. M. de Mul, and J. Greve, J. Raman Spectrosc. 22, 217 (1991).

18. G. J. Puppels, A. Huizinga, H. W. Krabbe, H. A. de Boer, G. Gijsbers, and F. F. M. de Mul, Rev. Sci. Instrum. 61, 3709 (1990). 